\title{
Examining Supports and Barriers to Breastfeeding through a Socio-Ecological Lens: A Qualitative Study
}

Kailey Snyder ( $\sim$ kaileysnyder@creighton.edu )

Creighton University School of Pharmacy and Health Professions https://orcid.org/0000-0003-1365-3556

Emily Hulse

Center for the Child \& Community

Holly Dingman

Center for the Community and Child

Angie Cantrell

MilkWorks LLC

Corrine Hanson

University of Nebraska Medical Center

Danae Dinkel

University of Nebraska at Omaha

\section{Research}

Keywords: Breastfeeding, Socioecological Model, Qualitative, Policy

Posted Date: February 9th, 2021

DOI: https://doi.org/10.21203/rs.3.rs-59627/v4

License: (c) (i) This work is licensed under a Creative Commons Attribution 4.0 International License. Read Full License

Version of Record: A version of this preprint was published at International Breastfeeding Journal on July 12 th, 2021. See the published version at https://doi.org/10.1186/s13006-021-00401-4. 


\section{Abstract}

BACKGROUND: Early breastfeeding cessation is a societal concern given the massive benefits associated with breastfeeding for mother and child. More effective interventions are needed to increase breastfeeding duration. Prior to developing such interventions more research is needed to examine breastfeeding supports and barriers from the perspective of breastfeeding stakeholders. One such framework that can be utilized is the Socio-Ecological Model which stems from Urie Broffenbrenner's early theoretical frameworks (1973-1979). The purpose of this study was to examine supports and barriers to breastfeeding across environmental systems.

METHODS: A total of 49 representatives participated in a telephonic interview. Interviewees represented various levels of the model based on their current breastfeeding experience (i.e., mother or significant other) or occupation. A direct content analysis was performed as well as a constant comparative analysis to determine differences between level representatives.

RESULTS: Common supports identified by all interviewees were in-hospital breastfeeding education (organizational level) and the existence of breastfeeding protection legislation (policy level). Barriers identified by all interviewees included a lack of support (interpersonal level), lack of hospital resources (organizational level) and lack of specificity within the existing breastfeeding protection legislation (policy level). Other identified supports and barriers varied by representatives for each level of the model.

CONCLUSION: Future efforts should target multiple levels of the SEM to eliminate the disparities between breastfeeding mothers' perceptions and the stakeholders working to increase breastfeeding initiation and duration rate.

\section{Introduction}

The short and long-term benefits of breastfeeding for child and mother are well-established [1]. Not only can breastfeeding support infant survival in the first year of life but it can also produce long-term benefits in intelligence, academic achievement and reduce risk for chronic conditions later in life [1-2]. Further, breastfeeding mothers have a lower risk of type 2 diabetes, hypertension, breast and ovarian cancer [3]. Despite the significant health benefits, breastfeeding rates are still well-below the recommended duration of exclusive breastfeeding for at least the first 6 months of life [4-8]. Research suggests that concurrent intervention delivery using a combination of systems such as home, family, healthcare and community involvement improves breastfeeding rates; however, few successful interventions are currently in practice [9]. One such theory that can be used to understand human development is Urie Brofenbrenner's bioecological theory [10].

Brofenbrenner's theoretical perspective has evolved greatly over time, however his early work (1973-1979) provides a strong foundation for understanding the complexities of engaging in a behavior like breastfeeding [11-15]. Per Brofenbrenner, "the ecology of human development involves the scientific study of the progressive, mutual accommodation between an active, growing human being and the changing properties of the immediate settings in which the developing person lives, as this process is affected by relations between these settings, and by the larger contexts within which the settings are embedded" (Pg. 21) [16]. Broffenbrenner posited there were four types of systems that could bidirectionally influence development (microsystem, mesosystem, exosystem and macrosystem). Important to this study, the microsystem was defined as the proximal setting where one can have individualized interactions (e.g., home, childcare, work, healthcare) [12]. A breastfeeding women's microsystem consists of many individuals that have the potential to influence her breastfeeding journey. For instance, her family 
and/or friends, her childcare providers, her healthcare providers and public health professionals. Despite the substantial influence these individuals may have on a breastfeeding woman's experience, few studies have attempted to understand the reciprocal interaction between mothers and individuals within their microsystem. Importantly, a comparison of perspectives of those occupying various microsystems would help to elucidate the proximal processes influencing a mother's breastfeeding journey [15].

Globally, Broffenbrenner's conceptual framework has been frequently adapted to help elucidate health promotion endeavors [10]. This framework is often identified as the Socio-Ecological Model (SEM) [10]. While the SEM has been utilized in health promotion research there are criticisms of inappropriate use associated with based on the evolution of Broffenbrenner's theory over his lifespan [17]. This study will focus primarily on interpreting Brofenbrenner's early theoretical perspective (1973-1979) [11-15]. The SEM holds that health behaviors are affected by the interaction between an individual, their community and their environment. Typically, spheres of individual, interpersonal, community, organizational and policy environments are considered. The first level, individual, includes items such as personal knowledge, attitude and behavior. The second level, interpersonal, includes formal and informal social support systems. This support typically stems from family, friends, peers and co-workers. The third level is the community level and focuses on how community organizations provide one another with formal and informal support. The fourth level, organization, focuses on rules and regulations that affect how services may be provided to an individual. Finally, the fifth level, policy, focuses on local, state, national and global policies tevet that can influence resource allocation and access [18].

Importantly, a women's breastfeeding journey can be impacted by factors at each level of the SEM. For example, research has shown factors such as low self-efficacy (individual), lack of partner support (interpersonal), community stigma (community), hospital formula samples (organizational) and lack of protective laws (policy), hinder breastfeeding [19-22]. Conversely, factors at each level have also been identified as breastfeeding supports such as high self-efficacy (individual), supportive family and friends (interpersonal), access to community resources (community), in-hospital education (organizational) and workplace protections (policy) [8, 23-25]. Furthermore, there are individuals within a breastfeeding women's microsystem that can influence each level of the SEM [17]. To the researchers' knowledge few studies have utilized SEM to explore breastfeeding behavior [26-27]. The studies that have been conducted were limited to the perspectives of mothers and healthcare providers. Research is needed to understand factors across SEM levels to understand how to best support women in their breastfeeding journey. Exploring the perspectives of individuals that directly interact within a women's microsystem and represent each level of the SEM is critical prior to further intervention development and to the researchers' knowledge has not been undertaken done before.

\section{Methods}

Design: A cross-sectional qualitative design guided by the SEM and grounded theory was utilized.

Setting: Participants were recruited throughout the state of Nebraska with an emphasis on achieving geographic diversity in the sample. Thus, recruitment methods targeted both rural and urban areas. Urban and rural areas were differentiated by census tract-based rural urban community area codes. Urban residents were defined as rural urban community area codes 1-6, and rural residents as codes 7-10 [28].

Sample: A purposive sampling technique was utilized as is typically required of grounded theory study [29]. Representatives of each level of the SEM were recruited on the basis of their profession orientation or breastfeeding history. Recruitment methods included e-mailing all current members of the State Breastfeeding Coalition, posting 
interview information on local and statewide breastfeeding Facebook support groups, and snowball sampling. Interested participants were encouraged to contact the first author to directly to set up a time to conduct an interview. A goal of 12 participants per level was sought, however, once ongoing data analysis indicated saturation within a level participant recruitment was halted. Those classified at the individual level were current breastfeeding mothers $(n=12)$. Participants at the interpersonal level were identified as personal supports to breastfeeding mothers. These included in-home childcare providers $(n=6)$ and partners of breastfeeding mothers $(n=4)$. Individuals representing the community level served as community leaders and advocates for breastfeeding (e.g., childcare center directors $(n=6)$, a peer lactation counselor $(n=1)$ certified lactation counselors $(n=3)$, social worker $(n=1)$ and a medical librarian/community advocate $(n=1))$. Those representing the organizational level served in an administrative capacity that had the capability to develop or alter rules and regulations for breastfeeding supports and services within an organization (i.e., community program administrators/managers $(\mathrm{n}=8)$ and maternal/child health nonprofit directors $(n=2)$ ). Finally, those representing the policy level were involved in policy development and decision-making related to breastfeeding (i.e., Health Department Division Chief $(n=1)$ ) or had expertise in the field (i.e., MD, IBCLC $(\mathrm{n}=4)$ ). The 4 healthcare providers interviewed had altbeen actively involved in Nebraska breastfeeding legislation within the past ten years through by providing written or in-person testimony.

Data Collection: A total of 49 telephonic semi-structured interviews were conducted between the months of May and August, 2019. Informed consent was obtained verbally as per the approval of a University-affiliated institutional review board. Participants were read a brief summary of the study purpose and risks, involved and told informed that their participation was voluntary and they had the right to withdraw at any time. Participant details such as age, race/ethnicity and occupation were documented. Each interview took approximately 25 minutes to complete.

The 15-question interview guide utilized a semi-structured format guided by the SEM. The interviews focused on participant perceptions on how various levels of the SEM supported or hindered breastfeeding. Questions were developed by a qualitative research expert and piloted with a stakeholder representing each of the five SEM levels. Face validity was conducted within these five pilot interviews to ensure the wording was clear and interpreted accurately [30]. These-pilot interviews were transcribed verbatim by a trained researcher and reviewed by a qualitative expert for accuracy of the transcription. Small wording changes $(n=15)$ were made for clarification and the interview process was considered complete.

Data Analysis: A framework for grounded theory analysis was utilized to enhance the validity of findings [29, 31]. All interviews were transcribed by the interviewer and reviewed for accuracy by the primary author. The first step in the analysis process was a direct content analysis. The direct content analysis focused on identifying preliminary coding categories and coding based on operational definitions of SEM constructs. Steps included two researchers reviewing all interview transcripts in their entirety twice. Two researchers separately identified and coded statements that directly related to one of the SEM levels. Both kept memo notes throughout the coding process and discussed discrepancies [32]. The next step involved a constant comparative analysis [29]. This analysis involved both researchers scrutinizing the responses of participants identified as representing each level of SEM to compare their experiences and identify categories of significance. This process facilitated the strategy of intuiting, that is, identification of themes identified in the participant's accounts [33]. This strategy resulted in a more in-depth analysis at each level of SEM based on the stakeholders' reflections. The themes produced across the cases were then categorized into subthemes under the initial themes identified. A further analysis of rural versus urban participants was also undertaken. 
A peer debriefing took place throughout the process of analysis [31]. A second qualitative researcher reviewed the themes identified following the direct content analysis and comparative analysis. Consensus was achieved through frequent discussion, and changes made until both authors reached consensus on the themes and subthemes.

\section{Results}

The mean age of the 49 participants was $38.7 \pm 10.1$. The majority $(85.7 \%)$ identified as Caucasian; $8.2 \%$ were African American, 4.1\% were Caucasian/Asian American, and a further $2.0 \%$ identified as Hispanic. The majority of participants $(75.5 \%)$ resided in an urban residence, with the remaining $24.5 \%$ residing in a rural area (see Table 1 ).

\section{Supports and Barriers to Breastfeeding}

Figures 1 and $2 \mathrm{~s}$ list the major themes identified at each level of the SEM. The stakeholders identifying each theme is denoted via a symbol. Figure 1 notes the most commonly reported themes for breastfeeding support among the interview sample. Figure 2 denotes the most common barriers to breastfeeding.

\section{Individual Factors}

At the individual level, under the theme 'breastfeeding support' the two main themes involved breastfeeding as a valued behavior, and a desire for mothers to try to breastfeed. Breastfeeding mothers (individual level), significant others (interpersonal level) and community representatives reported that they were seeing women personally valuing breastfeeding to a greater degree than in the past with mothers having a strong desire to "try" to breastfeed. For instance, a Community Health coordinator reported, "I think it is becoming more popular nowadays, to at least attempt to start breastfeeding. Women will brag that they made it [for] a whole year or breastfed six months."

Individual barriers were-typically related to time commitments, exhaustion and isolation. In terms of to the time commitment, participants representing individual, interpersonal and organizational levels most often reported this issue. For example, a labor and delivery nurse stated, "just the time commitment of it. I mean I always say it's not hard it's just demanding you to live on a two-hour clock." Exhaustion was also a common theme reported by those at the interpersonal, community and policy level. For instance, a one community program coordinator noted, "I think the lack of sleep that comes with a newborn. You know you're not well-rested and you're trying to have good mental health and it's a struggle." Moreover, all currently breastfeeding mothers reported isolation as an issue. One mother, for example, admitted, "I would definitely say like kind of the isolation factor of it. You're the only one who can do it and sometimes it's a little lonely just feeling stuck sometimes".

\section{Interpersonal Factors}

At the interpersonal level, social media, peer-to-peer and family were identified as the greatest sources of support. For example, a County Health Director stated, "I see a really strong social media presence, a supportive social media presence. It seems like women are going to social media to find support." General peer-to-peer support was also reported by interpersonal and community representatives. A husband of a breastfeeding women noted, "I think what really helped my wife was the support groups she found that allowed for mother-to mother peer counseling." Finally, familial support was often stated as a key influencer of breastfeeding support by those at the individual, community, organizational and policy level. A community program coordinator stated, "Some of the biggest support pieces that I feel like are critical are having support from your own family." 
The main barrier identified by all interviewed participants was related to a lack of support from family and/or friends. For example, a social worker stated: "I would say probably lack of social supports. A lot of our moms want to breastfeed and they don't have a lot of support from like dads or friends."

\section{Community Factors}

At the community level, representatives of the community, organization and policy level reported that normalization of breastfeeding was occurring to at least some degree. Representatives at the individual, interpersonal and organizational level reported ample access to community lactation support. When describing breastfeeding normalization, childcare provider stated, "I think it's becoming better, it's more socially normally to see a mother breastfeeding in public. I think it's not as shunned upon not to do it in public and everything." Related to community lactation support, many participants reported the existence of several community organizations or support groups. For example, a currently breastfeeding mother reported, "definitely places like [community breastfeeding non-profit] for lactation support...it's helpful I feel like just to have places like that in the community that women can go."

A lack of community resources in rural and underserved areas as well as a lack of normalization were identified are community level barriers.

Specific to the rural disparities, participants at the community, organizational and policy levels reported this most frequently. One nurse residing in a rural area reported, "we have very minimal support. When I moved here, I searched for support groups and there was nothing to be found." A lack of normalization was identified at the individual and interpersonal level. One mother noted, "It's just hard to breastfeed in public. I know it's supposed to be a thing you can do everywhere but sometimes it's just not really looked at as acceptable yet".

\section{Organization Factors}

At the organizational level, support was related to hospitals having helpful procedures in place regarding breastfeeding and effective in-hospital education directly after birth. Those at the community, organization and policy level typically reported the hospital procedures as supportive. For example, a home-visiting IBCLC stated, "I think they [hospitals] have done a great job with all of the new policies that we've put in place so the sacred hour, skin-to-skin, delaying the bath, they've put a lot of things in place to help breastfeeding moms."

Conversely, although not a majority, two healthcare providers statedthey worked in facilities in which mothers were given formula even prior to their child's birth. For example, a labor and delivery nurse residing in a rural area stated,

"They give out formula at your first visit when you come to the hospital to register before you come in for delivery. they send you home with a bunch of [formula brands]."

A lack of hospital resources despite good procedures was also cited. One example came from an IBCLC that stated, "It would be nice if they could have more CLC's or IBCLC's on staff because what I hear from families is that there was an IBCLC there but they weren't able to spend much time with them".

Policy Factors 
At the policy level, current laws and workplace protections were reported by the majority of participants. An IBCLC stated, "I think they [laws] have been very helpful, especially with moms going back to work, you know the laws to breastfeed in public and the pumping laws have definitely been a huge help".

Conversely, a majority of participants felt there was still a lack of specificity within the existing breastfeeding laws/policies that left women unprotected. A community program manager noted "I know there are policies and laws but I feel like some of those still have loopholes. Like it doesn't seem to cover every occupation especially those teachers and nurses who need varying pumping schedules."

\section{Discussion}

This qualitative inquiry took a unique investigative approach by utilizing the SEM and interviewing individuals within a breastfeeding women's microsystem to understand her breastfeeding supports and barriers [11-15]. Not only did this identify supports and barriers at each level of the SEM but it also highlighted disconnects between breastfeeding mothers and those they interact with. Focusing on these disconnects could improve maternal/child health efforts.

First, at the individual level, currently breastfeeding mothers reported isolation as a barrier while those representing the other SEM levels attributed individual barriers to be more related to time commitment or exhaustion. This suggests despite interaction with breastfeeding mothers their supporters may be unaware of feelings of isolation. This could be especially true during the COVID-19 pandemic. Related to the interpersonal level, mothers reported frequent use of social media. This is similar to previous research that have found mothers enjoy the immediate support and community that can be obtained through social media avenues [34]. Unfortunately, social media has also been associated with inaccurate education [35]. Thus to combat isolation, innovative support strategies using social media and mHealth technologies (e.g., mobile health applications) should continue to be improved [36].

Second, normalization of breastfeeding was identified at the community and organizational levels but a lack of normalization was reported at the individual and interpersonal levels. Despite policy and legislation improvements there is still an absence of images of women breastfeeding in community settings which could be driving the notion that breastfeeding is something to be done in solitude [37]. Further, this could be influencing perceptions of isolation

Third, while hospitals were applauded for the improvements made to procedures and breastfeeding education a scarcity of resources to maintain these improvements was identified. Bridging relationships between hospitals and local community effort could be beneficial. For example, hospitals could use community lactation providers to support in-hospital education classes or even follow-up support protocols (i.e., phone follow-ups). Further, healthcare workers in the hospital setting should be aware of all community resources available (i.e., La Leche Leagues, community facebook groups, community non-profits) and be able to effectively refer women to these resources. This could support the large drop in exclusive breastfeeding that is occurring within the first 2 weeks postpartum [38].

Fourth, while the legislation and policies in place to support breastfeeding mothers were recognized there was still a desire for greater specificity within these policies. Promisingly, research has shown mothers serving in government positions consistently produce the most legislative bills related to children and family [39]. Thus, now is an optimal time to contact working mothers that serve in political roles to share the identified needs of breastfeeding women related to more specific workplace protections and improved maternity leave protections worldwide. 


\section{Limitations}

This study was weakened by individuals being selected based on their breastfeeding relationship or employment as they may have also identified at other levels of the SEM. For instance, an IBCLC who had breastfed within the past 5 years may have greatly influenced her answers by her personal breastfeeding experience. There was an attempt to mitigate this limitation by asking individuals to answer based on their employment type however personal influences may have been impacted responses. In addition, this study was weakened by being limited to only one state in the Midwestern United States, further research is needed in other geographic locations to determine the generalizability of findings.

\section{Conclusion}

A mother's microsystem can greatly influence her breastfeeding journey. The findings from this study indicate four areas of opportunity that should be considered when developing future interventions to support breastfeeding mothers. These include; leveraging technologies to enhance maternal support opportunities, increasing public awareness of breastfeeding in the community, bridging relationships between community coalitions/organizations and hospitals and finally enhancing connections with politicians, especially mothers, in political roles. These strategies have the potential to enhance support within a woman's microsystem and reduce the barriers hindering her breastfeeding journey.

\section{Declarations}

Ethics approval and consent to participate: The Creighton University affiliated IRB approved this research. All participants provide verbal assent prior to participation.

Consent for publication: Not Applicable

Availability of data and materials: The datasets used and/or analyzed during the current study are available from the corresponding author on reasonable request.

Competing Interests: The authors declare that they have no competing interests.

Funding: There is no funding associated with this study.

Authors' Contributions: $\mathrm{KS}, \mathrm{EH}, \mathrm{HD}, \mathrm{AC}, \mathrm{CH}, \mathrm{DD}$ assisted in the development of the interview guide and participant recruitment. KS and DD analyzed the interview data. All authors assisted in writing the manuscript, KS was the main contributor. All authors read and approved the final manuscript.

Acknowledgements: The authors have no acknowledgements.

\section{References}

1. Horta, B. Breastfeeding: Investing in the future. Breastfeed Med. 2019;14;S1.

2. Schanler, R, Krebs, N, Mass, S. American Academy of Pediatrics, \& American College of Obstetricians and Gynecologists. Breastfeeding Handbook for Physicians, American Academy of Pediatrics. Elk Grove Village. $2^{\text {nd }}$ 2019; 107-134. 
3. Feltner C, Weber RP, Stuebe A, Grodensky CA, Orr C, Viswanathan M. Breastfeeding programs and policies, breastfeeding uptake, and maternal health outcomes in developed countries. Comp Effect Rev. 2018.

4. Beauregard JL, Hamner HC, Chen J, Avila-Rodriguez W, Elam-Evans LD, Perrine CG. Racial Disparities in breastfeeding initiation and duration among US infants born in 2015. Morb Mortal Wkly Rep. 2019; 68(34):745.

5. Dayton CJ, Johnson A, Hicks LM, Goletz J, Brown S, Primuse T, et al. Sex differences in the social ecology of breastfeeding: a mixed methods analysis of the breastfeeding views of expectant mothers and fathers in the US exposed to adversity. J.Biosoc. 2019;51(3):374-93.

6. Eidelman Al. Breastfeeding and the use of human milk: an analysis of the American Academy of Pediatrics 2012 Breastfeeding Policy Statement. Breastfeeding Med. 2012; 7(5):323-4.

7. Grubesic TH, Durbin KM. A spatial analysis of breastfeeding and breastfeeding support in the United States: the leaders and laggards landscape. J Hum Lact. 2019;35(4):790-800.

8. Snyder, K., Hansen, K., Brown, S., Portratz, A., White, K., \& Dinkel, D. Workplace breastfeeding support varies by employment type: the service workplace disadvantage. Breastfeeding Med. 2018; 13(1), 23-27.

9. Sinha B, Chowdhury R, Sankar MJ, Martines J, Taneja S, Mazumder S, et al. Interventions to improve breastfeeding outcomes: A systematic review and meta-analysis. Acta Paediatr. 2015; 104:114-34.

10. Kilanowski JF. Breadth of the socio-ecological model. J of Agromed. 2017; 22:4, 295-297.

11. Bronfenbrenner, U. Social ecology of human development. In F. Richardson (Ed.), Brain and intelligence: The ecology of child development; Hyattsville, MD: National Education Press. 1973 (p. 113-129).

12. Bronfenbrenner, U. Reality and research in the ecology of human development. Proc Am Philos Soc. 1975; 119, 439-469.

13. Bronfenbrenner, U. The experimental ecology of education. Teachers College Record. 1976; 78, 157-204.

14. McLeroy KR, Bibeau D, Steckler A, Glanz K. An ecological perspective on health promotion programs. Health Educ Q. 1988;15(4):351-77.

15. Module 1: What are the social ecological model (SEM), communication for development (C4D). 2016: https://www.unicef.org/media/47781/file/UNICEF_2017_Report_on_Communication_for_Development_C4D.pdf

16. Sayres S, Visentin L. Breastfeeding: uncovering barriers and offering solutions. Curr Opin Pediatr. 2018; 30(4):591-6.

17. Iliadou M, Lykeridou K, Prezerakos P, Swift EM, Tziaferi SG. Measuring the effectiveness of a midwife-led education programme in terms of breastfeeding knowledge and self-efficacy, attitudes towards breastfeeding, and perceived barriers of breastfeeding among pregnant women. Mater Sociomed. 2018; 30(4):240.

18. Rempel LA, Rempel JK, Moore KC. Relationships between types of father breastfeeding support and breastfeeding outcomes. Matern Child Nutr. 2017: 13(3):e12337.

19. Albrecht SA, Wang J, Spatz D. A call to action to address barriers to breastfeeding and lactation faced by student-mothers. Nurs Womens Health. 2017; 1;21(6):431-

20. Abbass-Dick, J, Brown, H, Jackson, K, Rempel, L., \& Dennis, C. Perinatal breastfeeding interventions including fathers/partners: A systematic review of the literature. Midwifery. 2019; 75:41-51

21. Brockway, M, Benzies, K, \& Hayden, K. Interventions to improve breastfeeding self-efficacy and resultant breastfeeding rates: A systematic review and meta-analysis. J Hum Lact. 2017; 33:3:486-499.

22. Munn AC, Newman SD, Mueller M, Phillips SM, Taylor SN. The impact in the United States of the baby-friendly hospital initiative on early infant health and breastfeeding outcomes. Breastfeeding Med. 2016;11(5):222-30. 
23. Bueno-Gutierrez D, Chantry C. Using the socio-ecological framework to determine breastfeeding obstacles in a low-income population in Tijuana, Mexico: healthcare services. Breastfeeding Med. 2015;10(2):124-31.

24. Dunn RL, Kalich KA, Fedrizzi R, Phillips S. Barriers and contributors to breastfeeding in WIC mothers: A social ecological perspective. Breastfeeding Med. 2015;10(10):493-501.

25. Jacobson LT, Twumasi-Ankrah P, Redmond ML, Ablah E, Hines RB, Johnston J, et al. Characteristics associated with breastfeeding behaviors among urban versus rural women enrolled in the Kansas WIC program. Matern Child Health J. 2015;19(4):828-39.

26. Chun Tie Y, Birks M, Francis K. Grounded theory research: A design framework for novice researchers. SAGE Open Med. 2019 Jan;7: 2050312118822927.

27. Belone, L. Community-based participatory research conceptual model: Community partner consultation and face validity. Qual Health Res. 2016; 26(1): 117-135.

28. Lincoln YS, Guba EG. Naturalistic inquiry (vol. 75). Sage Thousand Oaks. 1985.

29. Hsieh HF, Shannon SE. Three approaches to qualitative content analysis. Qual Health Res. 2005;15(9):1277-88.

30. Wojnar DM, Swanson KM. Phenomenology: an exploration. Journal of holistic nursing. 2007 Sep;25(3):17280.Ayres, L, Kavanaugh, K., \& Knafl, K. Within-case and across-case approaches to qualitative data analysis. Qual Health Res. 2003; 13:6: 871-883.

31. Bridges, N. The faces of breastfeeding support: Experiences of mothers seeking breastfeeding support online. Breastfeeding Rev. 2016. 24(1).

32. Snyder, K., Kneip Pelster., \& Dinkel, D. Healthy eating and physical activity among breastfeeding women: the role of misinformation. BMC Pregnancy Childbirth. 2020; 20:(1):1-7

33. Schindler-Ruwisch, J. M., Roess, A., Robert, R. C., Napolitano, M. A., \& Chiang, S. (2018). Social support for breastfeeding in the era of mHealth: a content analysis. J Hum Lact, 34(3), 543-555.

34. Giles F. Images of women breastfeeding in public: solitude and sociality in recent photographic portraiture. Int Breastfeed J. 2018; 13(1):1-2.

35. Brand E, Kothari C, Stark MA. Factors related to breastfeeding discontinuation between hospital discharge and 2 weeks postpartum. J Perinat Educ. 2011;20(1):36-44.

36. Bryant LA, Marin Hellwege J. Working Mothers Represent: How Children Affect the Legislative Agenda of Women in Congress. Am Politics Res. 2019;47(3):447-70.

\section{Table}

Table 1. Sociodemographics of Participants by SEM Level 


\begin{tabular}{|llllll|}
\hline Characteristics & $\begin{array}{c}\text { Individual } \\
(n=12)\end{array}$ & $\begin{array}{c}\text { Interpersonal } \\
(n=10)\end{array}$ & $\begin{array}{l}\text { Community } \\
(n=12)\end{array}$ & $\begin{array}{l}\text { Organizational } \\
(n=10)\end{array}$ & $\begin{array}{c}\text { Policy } \\
(n=5)\end{array}$ \\
\hline Age (Mean, SD) & $31.5 \pm 5.4$ & $36.0 \pm 6.3$ & $41.8 \pm 11.3$ & $42.1 \pm 11.0$ & $46.2 \pm$ \\
\hline Race/Ethnicity (n, \%) & & & & & 11.7 \\
\hline White & $11,91.6 \%$ & $9,90.0 \%$ & $10,83.3 \%$ & $10,100.0 \%$ & $4,80.0 \%$ \\
\hline African American & $1,8.3 \%$ & 0 & $1,8.3 \%$ & $0,0 \%$ & $1,20.0 \%$ \\
\hline Asian & $0,0 \%$ & $1,10.0 \%$ & $1,8.3 \%$ & $0,0 \%$ & $0,0 \%$ \\
\hline Hispanic & $0,0 \%$ & $1,10.0 \%$ & $2,16.6 \%$ & $0,0 \%$ & $0,0 \%$ \\
\hline $\begin{array}{l}\text { Geographic Residence }(\mathrm{n}, \\
\%)\end{array}$ & & & & & \\
\hline Rural & $2,16.6 \%$ & $3,30.0 \%$ & $4,33.3 \%$ & $3,30.0 \%$ & $0,0 \%$ \\
\hline Urban & $10,83.3 \%$ & $7,70.0 \%$ & $8,66.6 \%$ & $7,70.0 \%$ & $5,100.0 \%$ \\
\hline Employment type $(\mathrm{n}, \%)$ & & & & & \\
\hline Education Sector & $5,41.6 \%$ & $1,10.0 \%$ & $0,0 \%$ & $0,0 \%$ & $0,0 \%$ \\
\hline Health Sector & $1,8.3 \%$ & $6,60.0 \%$ & $9,75.0 \%$ & $8,80.0 \%$ & $5,100.0 \%$ \\
\hline Works inside the home & $4,33.3 \%$ & $0,0 \%$ & $0,0 \%$ & $0,0 \%$ & $0,0 \%$ \\
\hline Other & $2,16.6 \%$ & $3,30.0 \%$ & $3,25.0 \%$ & $2,20.0 \%$ & $0,0 \%$ \\
\hline
\end{tabular}

Figures 


\section{Breastfeeding supports}

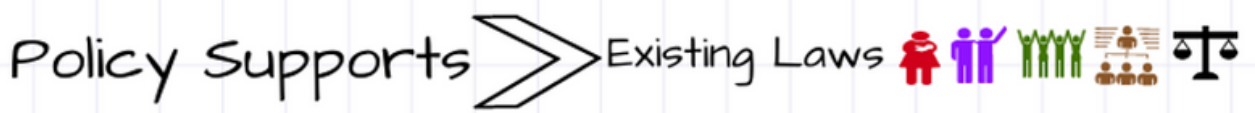

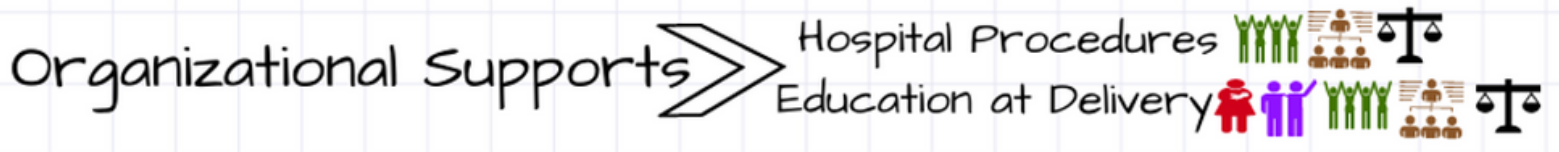

Community Supports Normalization is Happening Mtort of

Community Lactation Support 形 ili

Interpersonal Supports $\gg$ Peer to Peer inill mink

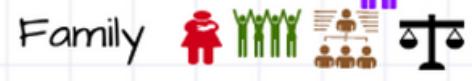

Individual Supports Breastfeeding is valued in iil /mmly

Desire to try

\section{Representatives}

î INDIDUAL iï INTERPERSONAL

MePfF COMMUNITY

JI POLICY

Figure 1

Breastfeeding Support Themes 


\section{Breastfeeding Barriers}

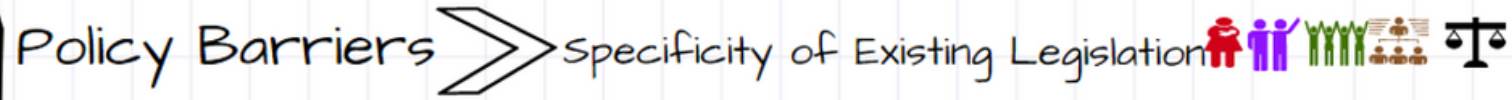

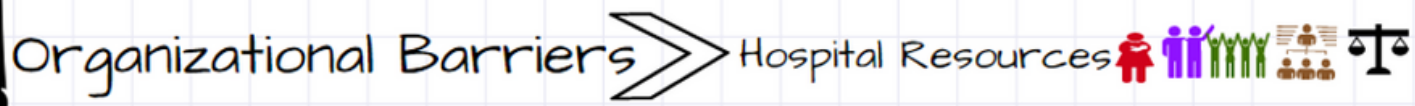

Community Barriers 》 Lack of Normalization

Interpersonal Barriers 8 Lack of support inilmm

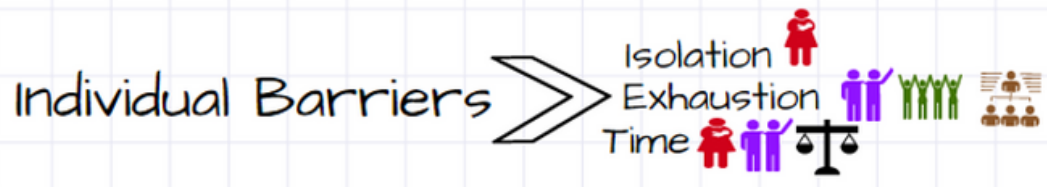

Representatives

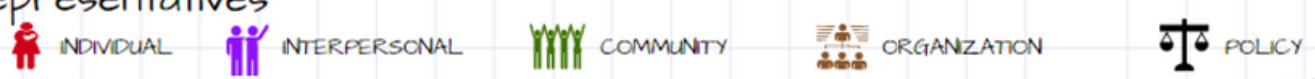

Figure 2

Breastfeeding Barrier Themes 